\title{
Temporal candidate gene expression patterns in the sow placenta during early gestation and the effect of maternal L-arginine supplementation
}

\author{
S. Novak', H.S. Moore', F. Paradis', G. Murdoch², M.K. Dyck', W.T. Dixon' \\ and G.R. Foxcroft ${ }^{1}$ \\ 'Swine Reproduction-Development Program, Dept. AFNS, University of Alberta, Edmonton, AB, \\ T6C 2P5, Canada; ${ }^{2}$ Department of Animal and Veterinary Science, College of Agricultural and Life \\ Sciences, University of Idaho, Moscow, ID, 83843-2330 USA
}

The trend towards high ovulation rates in mature commercial sows has resulted in intra-uterine crowding in the immediate post-implantation period, with negative impacts on placental development and later fetal development (Town et al. 2004). Factors that improve placental angiogenesis could offset the effects of intra-uterine crowding by supporting placental development at critical times in gestation. Feeding of L-arginine has been shown to have beneficial effects on placental vascularization in gilts (Hazeleger et al. 2007) and on litter size born in gilts (Mateo et al. 2007) and sows (Ramaekers et al. 2006). In the present study, we investigated the effects of $\mathrm{L}$-arginine supplementation to commercial sows on global placental gene expression, and on temporal changes in the expression of a panel of eight candidate genes known to be involved in angiogenesis, in early pregnancy.

Multiparous sows $(n-48$ ) were either non-supplemented Controls or were fed an L-arginine supplement (20g.d) from d 15 through 29 of gestation. A representative number of sows were euthanized on days 16 through 49 of gestation and embryonic and placental tissues were collected from two average-sized conceptuses from each uterine horn and placed in RNAlater for later analysis. To obtain temporal expression profiles for specific genes involved in placental angiogenesis, total placental RNA was extracted from all Control samples collected, reverse transcribed and real-time PCR used to determine the transcript abundance of: vascular endothelial growth factor (VEGF)-A; the two VEGF receptors, fms-related tyrosine kinase 1 (FLT1) and fetal liver kinase-1 (flk-1/KDR); hypoxia-inducible factor (HIF)1A; the Angiopoietins (ANGPT) -1 and -2 and their receptor, TEK tyrosine kinase; and finally Angiogenin (ANG)-1. The delta $\triangle \mathrm{C}$ t values were calculated using $18 \mathrm{~S}$ as an internal control, and data were analyzed using regression analysis (SAS Institute Inc., Cary, NC). To determine the cumulative effect of $\mathrm{L}$-arginine treatment, real-time PCR for these same candidate genes was also performed on the d 30 placental samples from both Control and L-arginine sows. The relative $\Delta C t$ values for $d 30$ samples were again calculated using $18 \mathrm{~S}$ as an internal control and data were analyzed using MIXED models (SAS Institute Inc., Cary, NC). Effects of L-arginine on global placental gene expression ( $n=4$ representative sows per treatment) were also analyzed using PigOligoArray slides and placental tissues collected at d 30 of gestation. Total RNA was extracted, purified using mRNA mini kits (Invitrogen), amplified with aminoallyl mRNA amplification kit (Ambion), and labeled with $\mathrm{Cy} 3$ or $\mathrm{Cy} 5$ in a random block dye-swap design. The hybridized slide images were captured with Genepix software and an Axon Scanner set for optimized PMT for each dye. Median spot intensities underwent Loess and quantile normalization and were analyzed using linear models, all in limma (Smyth, 2004). 
There were no differences in ovulation rate, embryo survival, live embryos, and embryo or placental weight between control and L-arginine treated sows at day 30 of gestation (Table 1).

Table 1. Reproductive Characteristics of the experimental sows at day 30 of gestation.

\begin{tabular}{lccc}
\hline Item & Control sows $(n-6)$ & L-arginine sows $(n-6)$ & P-value \\
\hline Parity & $4.0 \pm 0.35$ & $3.8 \pm 0.37$ & 0.698 \\
Ovulation Rate & $25.8 \pm 1.72$ & $23.2 \pm 1.80$ & 0.305 \\
Live Embryos & $15.9 \pm 1.14$ & $13.8 \pm 1.19$ & 0.216 \\
Embryo Survival (\%) & $64.1 \pm 4.6$ & $60.6 \pm 4.8$ & 0.603 \\
Embryo Weight at Day 30 & $1.98 \pm 0.11$ & $2.26 \pm 0.12$ & 0.105 \\
Placental weight at Day 30 & $30.60 \pm 3.66$ & $34.94 \pm 3.43$ & 0.402 \\
\hline
\end{tabular}

Temporal gene expression profiling in the Control samples revealed that angiogenin (ANG1) and VEGFA mRNA expression did not change $(P>0.05)$ from $d 17$ through 49 of gestation. However, mRNA expression increased $(P<0.0001)$ over early gestation for flk-1/KDR, a specific receptor for VEGFA, and for HIF1A, FLT1, TIE2, ANGPT1 and ANGPT2. Of these 8 genes, $\mathrm{L}$-arginine supplementation only affected placental ANG1 mRNA expression $(P=0.028)$ at $d$ 30 of gestation in treated sows compared to Control sows $(11.3 \pm 0.58$ vs $13.6 \pm 0.58)$. This is a promising result as ANG 1 was one gene whose expression did not change throughout early gestation in Control placental tissues. We are currently analyzing the effect of L-arginine on candidate gene expression at d 45 of pregnancy to determine if effects of L-arginine are more pronounced at later stages of gestation. The expression of ANG1 protein in d 45 placenta is also being investigated. Analysis of microarray data revealed that 60 genes were differentially expressed between Control and treated sows at an F-test p-value of $<0.01$. Only 5 genes showed higher expression in the Control sow placentae; whereas expression of other genes was higher in L-arginine supplemented sows (cell proliferation-inducing gene 15, RING finger protein 4, Apolipoprotein C-II, Retinol binding protein, and Progestin Receptor alpha).

Given the limited effects of $L$-arginine feeding on reproductive characteristics, these results suggest that there are other factors influencing placental development and uterine crowding which confound the possible beneficial effects of L-arginine supplementation. Multiparous sows can vary greatly in their ovulation rates, and may have much lower embryo survival rates by $\mathrm{d} 30$ of gestation compared to lower parity sows, and this variance may contribute to lack of consistent effects of treatment. Testing of L-arginine in gilts or first parity sows with similar uterine crowding in early gestation may more clearly define these effects. Nevertheless, evidence of higher expression of angiogenin 1 in L-arginine treated sows by real time PCR, and upregulation of multiple genes in the microarray analysis, confirms the potential for L-arginine to improve placental angiogenesis in early gestation.

\section{References}

Hazeleger W, Ramaekers P, Smits C \& Kemp B 2007. Influence of nutritional factors on placental growth and piglet imprinting. Pp 309-328 in Paradigms in Pig Science, J.Wiseman, M.A. Varley, S, McOrist and B. Kemp, eds., Nottingham Univ. Press, Nottingham.

Mateo RD, Wu G, Bazer FW, Park JC, Shinzato I \& Kim SW 2007 Dietary L-arginine supplementation enhances the reproductive performance of gilts. Journal of Nutrition 137 652-656.

Ramaekers P, Kemp B \& van der Lende T 2006. Progenos in sows increases number of piglets born. Journal of Animal Science 84 (Suppl. 1) 462 (Abstr.)
Smyth GK. 2004 Linear models and empirical bayes methods for assessing differential expression in microarray experiments. Stat Appl Cenet Mol Biol 3: Article 3.

Town SC, Putman CT, Turchinsky NJ, Dixon WT \& Foxcroft GR 2004 Number of conceptuses in utero affects porcine fetal muscle development. Reproduction 128 443-454. 